\title{
Carlos Poblete*
}

\section{Vesna Mandakovic*}

\section{EL ROL DE LA INMIGRACIÓN Y LAS EXPECTATIVAS DE CRECIMIENTO EN EL EMPRENDIMIENTO INNOVADOR: EL CASO DE CHILE}

La relación entre inmigración y emprendimiento ha sido estudiada principalmente en economías desarrolladas, sin embargo, como fenómeno es cada vez más común en los países emergentes como Chile. Este estudio analiza el vínculo entre las expectativas de crecimiento, la inmigración y el desarrollo de emprendimiento innovador. Usando una muestra de más de 6.800 individuos se ha identificado que la probabilidad de desarrollar emprendimientos innovadores es significativamente mayor cuando se es inmigrante y se tienen altas expectativas de crecimiento. Estos resultados ponen de relieve las características que pueden estar asociadas con el tipo de estrategias elegidas por los emprendedores sobre sus negocios.

Palabras clave: emprendimiento, empresa emergente, innovación, migraciones.

Clasificación JEL: J15, L26.

1. Introducción

El vínculo entre migraciones y emprendimiento ha sido abordado en la literatura básicamente desde la perspectiva de los países desarrollados. Casos emblemáticos como el de Silicon Valley, donde más de un 16 por 100 de los emprendimientos tecnológicos exitosos que se desarrollaron fueron por equipos liderados fundamentalmente por extranjeros, que atrajeron la atención sobre el emprendimiento y las

\footnotetext{
* Universidad del Desarrollo. Santiago de Chile.
}

migraciones (Saxenian, 1999; Hart y Acs, 2011). La evidencia internacional señala que en países desarrollados los inmigrantes tienen una mayor incidencia en el porcentaje de trabajadores por cuenta propia. En Estados Unidos los inmigrantes representan una parte importante de la fuerza trabajadora independiente (Kochhar, 2015) y poseen altas tasas relativas de tenencia de negocios en distintos sectores (Saxenian, 1999; Stephan y Levin, 2001; Wadhwa et al., 2007, 2008; Monti et al., 2007; Hart y Acs, 2011). Por otra parte, los extranjeros no solo tienen una mayor presencia en el mercado emprendedor, sino que 
además sus negocios reportan un mejor desempeño (Hart y Acs, 2011; Stephan y Levin, 2001; Neville et al., 2014).

Existe menos información sobre los emprendedores inmigrantes en países emergentes como Chile, donde el fenómeno es más reciente. De hecho, en los últimos diez años se ha experimentado una fuerte alza en el flujo de inmigrantes. Según el Departamento de Extranjería y Migración (2015), en Chile la población migrante ha pasado de representar un 0,8 por 100 de la población total en 1992 a un 2,3 por 100 en 2014, lo que suma cerca de 411.000 inmigrantes. Aun cuando el porcentaje de la población extranjera sobre el total nacional es menor que en el caso de países de larga tradición migratoria (aproximadamente un 11,3 por 100), Chile ha más que triplicado el stock de personas inmigrantes entre 1990 y 2015, lo que hace que el fenómeno migratorio se haya transformado en un desafío desde el punto de vista de las políticas públicas.

Chile es un país receptor de flujos migratorios provenientes principalmente de países vecinos, quienes pueden aspirar a una mejor calidad de vida que en sus países de origen, además de seguridad y estabilidad institucional. Considerando que el emprendimiento es una herramienta de integración al mercado laboral, es relevante analizar la calidad y el tipo de actividades emprendedoras que se están realizando.

En el contexto de una economía en desarrollo, la incorporación de innovación en la creación de nuevos negocios es una señal de emprendimientos de mayor impacto. Dado que la innovación es clave para el crecimiento y el desarrollo de las pequeñas empresas, puede esperarse que la innovación esté positivamente relacionada con la ambición del crecimiento (Terjesen y Szerb, 2008). Según Verhuel y Van Mil (2011), esto no solo es cierto para la innovación de productos, sino también para la innovación de procesos (el uso de una nueva tecnología para producir el producto/servicio).
En este estudio trataremos de analizar las características de los nuevos negocios que desarrollan los inmigrantes, específicamente qué tipo de empresas crean. Para esto hemos definido los negocios en función de si éstos innovan o no. Usando la metodología de Koellinger (2008), se clasificaron los emprendimientos en innovadores o imitadores a partir de tres criterios: novedad para los clientes, intensidad de la competencia directa y la utilización de tecnología. Utilizamos los datos recolectados en el Global Entrepreneurship Monitor (GEM) del año 2016, que encuesta a más de 6.800 individuos mayores de 18 años y estimamos la probabilidad de desarrollar un emprendimiento innovador en función del estatus de inmigrante y las expectativas de crecimiento.

El artículo se organiza de la siguiente manera. El apartado 2 introduce el marco teórico en el que se presenta una revisión de la literatura, primeramente, dando una breve reseña del ecosistema emprendedor en Chile, y luego en torno a la relación entre la innovación y la inmigración, y las expectativas de crecimiento. En el apartado 3 se presenta la metodología y descripción de las variables. El apartado 4 se centra en los resultados obtenidos, y en el último apartado presentamos las implicaciones y conclusiones del estudio.

\section{Marco teórico}

\section{Ecosistema de emprendimiento en Chile}

Las oportunidades que ofrece el mercado laboral del país de destino, son una de las razones que inciden en la decisión de emigrar de los individuos. Una de las alternativas laborales es realizar una actividad emprendedora y desarrollar sus propios negocios. Al respecto, algunos estudios señalan las robustas condiciones de contexto para emprender en Chile, posicionándolo en un lugar de vanguardia entre los países de la región, e incluso también a un nivel más global (Mandakovic y Serey, 2017). 
Chile ha realizado esfuerzos en los últimos diez años por generar un ecosistema que fomente el emprendimiento y la innovación, buscando atraer a emprendedores de alto impacto desde distintos lugares del mundo con programas como Start-up Chile. Recientemente se creó un programa denominado Visa-Tech, el cual consiste en facilitar el otorgamiento de visas de capital humano avanzado en áreas de servicios tecnológicos, tanto para la contratación de trabajadores dependientes como para la creación de empresas en esta área. Este permiso se enfoca en aquellas empresas y organizaciones asociadas al Ministerio de Economía; lo particular de este programa es que las solicitudes de visas deben ser resueltas en un plazo de 15 días, lo que facilita el inicio de actividades de las personas solicitantes.

\section{Inmigración e innovación}

En términos generales, diversas razones sugieren que existe una relación positiva entre la inmigración y la innovación (Hunt et al., 2010; Ozgen et al., 2011; Kerr, 2013). Por ejemplo, diversos estudios sugieren que la inmigración tiene el potencial de cambiar la composición demográfica y de habilidad de la fuerza de trabajo, de manera que pueda promover actividades innovadoras. Específicamente, Ozgen et al. (2011) sugieren que la inmigración contribuye al desarrollo de las ciudades a través de las aglomeraciones, las cuales a su vez benefician la innovación y el crecimiento (Audretch, 1998; Gordon y McCann, 2005; Kerr, 2010). Esto se daría porque estos cambios generados por la inmigración incentivan a las empresas a ubicarse en zonas aglomeradas, y de esta manera responder eficientemente frente a los rápidos avances en las tecnologías que reducen drásticamente los ciclos de vida del producto, aumentando el ritmo de evolución de los productos y servicios.

Un segundo factor está relacionado con el hecho de que una afluencia de inmigrantes también puede representar una amplia gama de ocupaciones, generando un proceso de autoselección de los inmigrantes en términos de habilidades, capacidades, riesgo y espíritu emprendedor, que podría influir positivamente en la innovación (Poot, 2008). Esto mejora tanto las habilidades disponibles como las necesidades laborales, reduciendo los costes de transacción, ya que la heterogeneidad en la disponibilidad de competencias en el mercado de trabajo permite disminuir el coste de búsqueda de ciertas habilidades específicas. Por ejemplo, la economía local puede beneficiarse de un flujo de inmigrantes altamente cualificados, como en el caso de la llegada de investigadores extranjeros, que puede considerarse un factor innovador clave (Chellaraj et al., 2005).

Los inmigrantes pueden traer diferentes tipos de conocimiento de los disponibles en la población local. Este vínculo estaría dado por las ideas que se transmiten entre las personas con diferentes conjuntos de información, y la innovación como un producto del conocimiento (Maré et al., 2010). Los inmigrantes pueden de esta forma aumentar la diversidad de conocimientos en un área específica $y$, a través de interacciones con las personas locales, contribuir a la innovación (Alesina y La Ferrara, 2005).

Finalmente, un tercer factor está dado porque la diversidad no solo se aplica en términos ocupacionales, sino también en la diversidad cultural. Al respecto, la evidencia (por ejemplo Jacobs, 1961, 1969) sugiere que las ciudades más innovadoras y prósperas se caracterizan por una gran diversidad étnica y cultural. Bajo esta perspectiva, la inmigración impulsaría la generación de economías más complejas, eficientes y dinámicas, incentivando a las nuevas empresas al desarrollo de comportamientos más innovadores para capturar esta diversidad. Al respecto, Stuen et al. (2012) destacan que la mano de obra extranjera es un importante insumo para la producción de nuevas ideas y productos. La inmigración es por lo tanto un motor del crecimiento y la competitividad, por lo que la política de inmigración está intrínsecamente ligada a la política de innovación. 


\section{Innovación y expectativas de crecimiento}

La literatura en emprendimiento ha explorado ampliamente las relaciones complejas entre la innovación y el desempeño de la empresa (Kalleberg y Leicht, 1991; Greve y Taylor, 2000; Klomp y Van Leeuwen, 2001), los procesos de creación de empresas y el conocimiento de la empresa y los trabajadores (Dewar y Dutton, 1986; Amason et al., 2006). La innovación se considera no solo como un ingrediente esencial del éxito financiero de los nuevos negocios (Ireland y Webb, 2007; Baron y Tang, 2011), sino también como uno de los factores clave del crecimiento de las pequeñas empresas (Heunks, 1998; Rauch y Frese, 2007; Rosenbusch et al., 2011). Por ejemplo, Stuart (2000) indica que la capacidad de innovar, adoptar nuevas tecnologías y aplicarlas a las condiciones locales es crucial para aumentar la productividad de las pequeñas y medianas empresas. En términos comparativos, se ha observado que las innovaciones radicales tienen un impacto grande y positivo en los beneficios de la empresa, mientras que las innovaciones incrementales tienen un impacto positivo en los ingresos, pero no necesariamente aportan mucho en los beneficios (Kim y Mauborgne,1997). Sin embargo, los riesgos y la incertidumbre en el desarrollo de una innovación tan radical son también mayores y, por su naturaleza global, la innovación radical no es tan común como otros tipos de innovación (Kim y Mauborgne,1997).

Con independencia de las características específicas de la innovación, inherentemente, una empresa que desarrolla innovación tendrá que tomar decisiones y crear sus propios mercados al tiempo que se enfrenta a una limitada disponibilidad de información, una alta incertidumbre y riesgos. Es por eso que el coste que implica innovar está justificado por una mayor ambición de los emprendedores con sus negocios (Aghion et al., 2001). Así, el nivel de innovación de una empresa no solo está a menudo relacionado con el tamaño de ésta, sino también con las expectativas de crecimiento (Stam y Wennberg, 2009). De hecho, Gundry y Welsch (2001) encuentran que los empresarios orientados al crecimiento son más propensos a seguir el cambio tecnológico que otros empresarios.

La innovación es uno de los instrumentos fundamentales de las estrategias de crecimiento para entrar en nuevos mercados (Gunday et al., 2011). Las innovaciones proporcionan a las empresas una orientación estratégica para superar los problemas que encuentran mientras se esfuerzan por lograr una ventaja competitiva sostenible (Drucker, 1985; Kuratko et al., 2005). Al innovar se obtienen ventajas competitivas y potencialmente una mayor participación en el mercado, contribuyendo indirectamente también a una mejor y más sostenible reputación. Gunday et al. (2011) señalan que una de las principales razones de innovar es precisamente el deseo de las empresas de obtener un mayor rendimiento empresarial y una mayor ventaja competitiva.

\section{Metodología}

\section{Muestra}

Usamos como principal fuente de información los datos del Global Entrepreneurship Monitor correspondiente al año 2016. Los datos recogidos entre la población adulta se utilizan para producir indicadores que miden la actividad emprendedora, las actitudes y las aspiraciones de los individuos. El informe de ese año en particular incluye un módulo especial sobre emprendimiento transnacional (Amorós et al., 2016), por lo que fue posible establecer el lugar de nacimiento de los individuos encuestados, además de los indicadores habituales de la encuesta. EI GEM en Chile tiene la particularidad de que es representativo a nivel regional, lo que permite usar condiciones de contexto de cada una de las regiones en las que se genera actividad emprendedora. Se trabajó con una muestra de más de 6.800 individuos mayores de 18 años.

Adicionalmente, con la finalidad de describir el contexto en el que se desarrolla la actividad emprendedora 
de los individuos, se utilizaron dos fuentes adicionales de información: del Departamento de Extranjería y Migraciones (DEM) y del Instituto Nacional de Estadística (INE).

\section{Variable dependiente, independiente, controles}

Lo que intentamos explicar a través de este estudio es la probabilidad de que un individuo sea un emprendedor «innovador». La definición de un emprendedor innovador proviene del trabajo realizado por Koellinger (2008). Koellinger también usa el GEM para construir la variable dependiente. Particularmente hay tres preguntas asociadas a innovación en procesos (usos de nuevas tecnologías) y producto (pocos competidores o novedad del producto para consumidores). En este caso la definición de la variable dependiente es un indicador binario que toma el valor uno si el emprendedor es innovador, y cero en caso contrario (Innov).

La principal variable independiente es el estatus de migrante que declara el individuo encuestado. Si el individuo nació fuera de Chile es considerado un migrante y por lo tanto la variable tomará el valor uno, en tanto que si el individuo nació en Chile la variable tomará el valor cero (Inmigrante).

La segunda variable dependiente de interés es la que refleja las expectativas de crecimiento en términos del número de empleados que pretende tener el emprendedor. Esta variable tomará el valor uno si el individuo declara que espera en los próximos cinco años un crecimiento del número de empleados superior a diez y de más del 50 por 100 en su cifra de negocios. Se considera como un indicador de la proporción de nuevos negocios con un alto potencial esperado de crecimiento (AltExpCrec).

Además, se controló por diversas características de los emprendedores, denotadas por $Z_{j}$ en la Ecuación 1. Esas variables de control son: la edad, si es mujer, educación universitaria, desempleado, capital social, capacidad de reconocer oportunidades, autoeficacia, temor al fracaso y si es un exportador. También se utilizan controles a nivel regional, tomando en consideración la heterogeneidad de la actividad empresarial en Chile, denotadas por $Z_{j}$ en la Ecuación 1. Esas variables son: la participación del producto interno de la región $j$ sobre el producto interno bruto del país (porcentaje de participación en el PIB) y el porcentaje de la población inmigrante en la región $j$ sobre el total de los habitantes de la región (porcentaje de población inmigrante). En el Cuadro 1 se presentan las estadísticas descriptivas de todas las variables anteriormente expuestas.

\section{Modelo empírico}

Dado que tenemos dos niveles de análisis, el primer nivel son las características y percepciones de los individuos y el segundo nivel corresponde a características de la región, utilizamos un modelo jerárquico o multinivel (Rabe-Hesketh y Skrondal, 2006; Aguinis et al., 2013). Autio et al. (2013) sugieren utilizar estos modelos de multinivel ya que se obtienen estimaciones más precisas, especialmente al usar los datos del GEM, donde la decisión de emprender no depende únicamente de las características y atributos de los individuos, sino también del contexto en el que se encuentren. El modelo a estimar es el que se presenta en la siguiente ecuación:

$$
\begin{gathered}
\operatorname{Pr}\left(\text { Innov }_{i j}=1\right)=\beta_{0}+\beta_{1} \text { Inmigrante }+\beta_{i}{ }_{2} \text { AltasExpCrec }_{j} \\
+\beta_{(3-12)} \text { Controles_indiv }{ }_{i}+\beta_{(13-14)} \text { Controles_region }_{j}+\mu_{i j}+\varepsilon_{j}
\end{gathered}
$$

Donde la obtención de una estimación positiva y significativa de $\beta_{1}$ implica que los inmigrantes tienen una mayor probabilidad de ser emprendedores innovadores que los individuos locales.

\section{Resultados}

El Cuadro 2 presenta los coeficientes de correlación parcial de las variables que utilizamos en la estimación. 


\begin{tabular}{|c|c|c|c|c|c|}
\hline \multicolumn{6}{|c|}{$\begin{array}{c}\text { CUADRO } 1 \\
\text { ESTADÍSTICAS DESCRIPTIVAS }\end{array}$} \\
\hline Variable & Obs. & Media & Desv. estándar & Min & Max \\
\hline Innov & 6.884 & 0,12 & 0,324 & 0 & 1 \\
\hline Mujer. & 6.884 & 0,49 & 0,500 & 0 & 1 \\
\hline 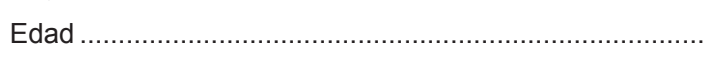 & 6.884 & 43,55 & 16,191 & 18 & 90 \\
\hline 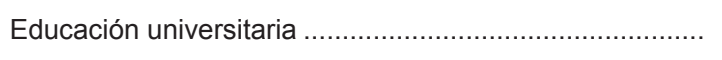 & 6.884 & 0,30 & 0,459 & 0 & 1 \\
\hline Desempleado & 6.884 & 0,10 & 0,301 & 0 & 1 \\
\hline Inmigrante & 6.884 & 0,02 & 0,150 & 0 & 1 \\
\hline Capital social & 6.884 & 0,42 & 0,494 & 0 & 1 \\
\hline 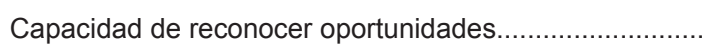 & 6.884 & 0,47 & 0,499 & 0 & 1 \\
\hline Autoeficacia & 6.884 & 0,64 & 0,480 & 0 & 1 \\
\hline Temor al fracaso & 6.884 & 0,31 & 0,461 & 0 & 1 \\
\hline Exportador & 6.884 & 0,04 & 0,186 & 0 & 1 \\
\hline 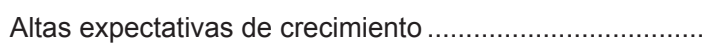 & 6.884 & 0,06 & 0,241 & 0 & 1 \\
\hline \% población inmigrante & 6.884 & 2,23 & 1,871 & 0,5 & 7,4 \\
\hline 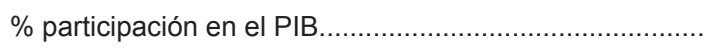 & 6.884 & 9,34 & 13,767 & 0,5 & 42,1 \\
\hline
\end{tabular}

Pero sí observamos que la variable Innov tiene una correlación positiva con la condición de inmigrante y positiva también con la variable que mide las altas expectativas de crecimiento de los emprendedores.

El Cuadro 3 presenta los resultados de las estimaciones correspondientes a la Ecuación 1. Las primeras cuatro columnas tienen las estimaciones realizadas con una regresión logística, en las que paulatinamente se fueron incorporando los controles a nivel individual, regional y las variables independientes. Se observa estabilidad en los coeficientes, lo que habla de la robustez del modelo presentado. La columna cinco presenta el modelo que se estima con el multinivel, que utilizamos dado que observamos que efectivamente la varianza entre las regiones era distinta de cero, lo que significa que además de la constante compartida por todas las regiones hay un efecto aleatorio asociado a cada región. Esto lo testeamos estimando el modelo solo con los efectos regionales, donde la hipótesis nula era que la varianza entre regiones era distinta de cero ${ }^{1}$.

Los resultados indican que variables relacionadas con las percepciones del emprendedor, como la capacidad de reconocer oportunidades, creencia de poseer conocimientos y habilidades para emprender (autoeficacia), así también como la presencia de capital social, juegan un rol importante en influenciar el desarrollo de emprendimientos innovadores. De igual manera, es posible evidenciar una relación positiva entre la generación de emprendimientos e innovaciones con el apuntar a mercados internacionales.

Por otra parte, y controlando por factores que habitualmente se ha visto que inciden en la probabilidad de que un individuo se declare un emprendedor innovador, la condición de inmigrante aumenta la probabilidad del emprender innovador. El coeficiente resulta

\footnotetext{
1 The test statistic is chibar2(01) $=29,57$ Prob $>=$ chibar2 $=0,0000$.
} 


\begin{tabular}{|c|c|c|c|c|c|c|c|c|c|c|c|c|c|c|}
\hline & & & CORR & $\begin{array}{r}\text { CU } \\
\text { RELA }\end{array}$ & $\begin{array}{l}\text { ADRC } \\
\text { CIÓN }\end{array}$ & $\begin{array}{l}02 \\
\text { PAR }\end{array}$ & CIAL & & & & & & & \\
\hline & 1 & 2 & 3 & 4 & 5 & 6 & 7 & 8 & 9 & 10 & 11 & 12 & 13 & 14 \\
\hline 1 Innov & 1 & & & & & & & & & & & & & \\
\hline 2 Inmigrante & 0,0542 & 1 & & & & & & & & & & & & \\
\hline 3 Mujer & $-0,0485$ & 0,0065 & 1 & & & & & & & & & & & \\
\hline 4 Edad & $-0,1165$ & $-0,0515$ & 0,0297 & & & & & & & & & & & \\
\hline 5 Educación universitaria & $-0,0047$ & 0,013 & $-0,0692$ & 0,0043 & 1 & & & & & & & & & \\
\hline 6 Desempleado & $-0,0565$ & $-0,0385$ & 0,173 & $-0,0521$ & $-0,1054$ & 1 & & & & & & & & \\
\hline 7 Capital social & 0,1214 & 0,0179 & $-0,0476-$ & $-0,0955$ & 0,1206 & $-0,0832$ & 1 & & & & & & & \\
\hline 8 Cap. reconocer oportunidades & 0,0695 & 0,0413 & $-0,0404-$ & $4-0,0944$ & $-0,0372$ & $-0,0303$ & 0,0828 & 1 & & & & & & \\
\hline 9 Autoeficacia & 0,1704 & 0,0324 & $-0,114$ & $-0,0252$ & 0,0726 & $-0,0664$ & 0,2162 & 0,0799 & 1 & & & & & \\
\hline 10 Temor al fracaso & $-0,0754$ & $-0,0176$ & 0,0519 & 0,0817 & 0,0186 & 0,017 & $-0,0677$ & $-0,1171$ & $-0,1867$ & 1 & & & & \\
\hline 11 Exportador & 0,2011 & 0,0539 & $-0,046-$ & $-0,0466$ & 0,0089 & $-0,0232$ & 0,0718 & 0,0512 & $0,1011-c$ & $-0,0552$ & 1 & & & \\
\hline 12 Alta exp. crecimiento & 0,2778 & 0,0171 & $-0,1218-$ & $3-0,0719$ & 0,0491 & $-0,0499$ & 0,1002 & 0,0516 & $0,1337-($ & $-0,0761$ & 0,2134 & 1 & & \\
\hline $13 \%$ población inmigrante & 0,0516 & 0,1057 & $-0,0017$ & 0,0111 & 0,0095 & $-0,0087$ & $-0,0084$ & 0,0023 & $0,0154-($ & $-0,0173$ & 0,055 & 0,0325 & 1 & \\
\hline 14 \% participación en PIB & $-0,0409$ & 0,0244 & 0,017 & 0,0183 & 0,0587 & 0,0093 & $-0,0467$ & $-0,0095-$ & $-0,0719$ & 0,0108 & $-0,0167$ & 0,0052 & 0,2854 & 1 \\
\hline FUENTE: Elaboración propia. & & & & & & & & & & & & & & \\
\hline
\end{tabular}

positivo y significativo $(\beta=0,431, p<0,1)$. También de manera consistente con la literatura encontramos que los individuos con mayor probabilidad de ser innovadores presentan altas expectativas de crecimiento $(\beta=1,631, p<0,01)$ y tienden a crear empresas que nacen con una visión internacional $(\beta=1,231, p<0,01)$. En el caso de un mercado pequeño como el chileno, que el emprendedor apunte a mercados internacionales es también una señal del potencial de crecimiento que tiene la empresa, que hace que al enfrentarse a una mayor competencia deba implementar una estrategia de innovación en procesos y productos.

Con respecto a la edad, observamos que es más probable que el individuo sea un emprendedor innovador si es más joven, conoce a otros emprendedores y no tiene educación universitaria completa. El Gráfico 1 presenta la probabilidad estimada de que el individuo sea un emprendedor innovador en relación a la edad, diferenciando entre el estatus de inmigrante y local. Observamos claramente que existe una relación negativa entre la edad y la probabilidad de emprender de manera innovadora, $y$ que esta probabilidad decreciente es superior en el caso de que el agente sea inmigrante. La edad promedio en la que los individuos emprenden en Chile es de 39 años; a esta edad la probabilidad del emprender innovador, si el individuo es inmigrante es de un 22 por 100 frente a un local cuya probabilidad asciende a un 9 por 100. A medida que la edad progresa, la diferencia entre locales e inmigrantes se va minimizando.

$\mathrm{Al}$ analizar las variables a nivel regional, observamos que una región que atrae a más población inmigrante tiene más probabilidad de escoger a emprendedores innovadores, y también se observa que un aumento en el producto interno relativo genera una disminución en la probabilidad de que los individuos generen actividades emprendedoras innovadoras. 
CUADRO 3

ESTIMACIÓN DE MODELO EMPÍRICO

\begin{tabular}{|c|c|c|c|c|c|}
\hline Variables & $\begin{array}{c}1 \\
\text { Logit } \\
\text { Innovador }\end{array}$ & $\begin{array}{c}2 \\
\text { Logit } \\
\text { Innovador }\end{array}$ & $\begin{array}{c}3 \\
\text { Logit } \\
\text { Innovador }\end{array}$ & $\begin{array}{c}4 \\
\text { Logit } \\
\text { Innovador }\end{array}$ & $\begin{array}{c}5 \\
\text { Multinivel } \\
\text { Innovador }\end{array}$ \\
\hline \multicolumn{6}{|l|}{ Nivel Individual } \\
\hline Mujer ............. & $\begin{array}{l}-0,0882 \\
(0,0795)\end{array}$ & $\begin{array}{l}-0,0907 \\
(0,0796)\end{array}$ & $\begin{array}{l}-0,0947 \\
(0,0798)\end{array}$ & $\begin{array}{c}0,0485 \\
(0,0828)\end{array}$ & $\begin{array}{c}0,0479 \\
(0,0831)\end{array}$ \\
\hline Edad & $\begin{array}{l}-0,0263^{* * *} \\
(0,00264)\end{array}$ & $\begin{array}{l}-0,0267^{* * *} \\
(0,00268)\end{array}$ & $\begin{array}{l}-0,0264^{* * *} \\
(0,00268)\end{array}$ & $\begin{array}{l}-0,0251^{* * *} \\
(0,00291)\end{array}$ & $\begin{array}{l}-0,0250^{* * *} \\
(0,00293)\end{array}$ \\
\hline Educación universitaria ....................... & $\begin{array}{l}-0,154^{*} \\
(0,0865)\end{array}$ & $\begin{array}{l}-0,138 \\
(0,0868)\end{array}$ & $\begin{array}{l}-0,137 \\
(0,0868)\end{array}$ & $\begin{array}{l}-0,198^{* *} \\
(0,0898)\end{array}$ & $\begin{array}{l}-0,181^{* *} \\
(0,0905)\end{array}$ \\
\hline Desempleado ................................... & $\begin{array}{l}-0,639^{* * *} \\
(0,167)\end{array}$ & $\begin{array}{l}-0,632^{* * *} \\
(0,167)\end{array}$ & $\begin{array}{l}-0,621^{* * *} \\
(0,168)\end{array}$ & $\begin{array}{l}-0,587^{* * *} \\
(0,169)\end{array}$ & $\begin{array}{l}-0,583^{* * *} \\
(0,169)\end{array}$ \\
\hline Capital social ...................................... & $\begin{array}{c}0,450^{* * *} \\
(0,0803)\end{array}$ & $\begin{array}{c}0,443^{* * *} \\
(0,0806)\end{array}$ & $\begin{array}{l}0,443^{* * *} \\
(0,0807)\end{array}$ & $\begin{array}{c}0,396^{* * *} \\
(0,0826)\end{array}$ & $\begin{array}{c}0,396^{* * *} \\
(0,0829)\end{array}$ \\
\hline Capac. reconocer oportunidades ........ & $\begin{array}{c}0,187^{* *} \\
(0,0794)\end{array}$ & $\begin{array}{c}0,189^{* *} \\
(0,0797)\end{array}$ & $\begin{array}{c}0,185^{\star *} \\
(0,0798)\end{array}$ & $\begin{array}{c}0,165^{\star *} \\
(0,0816)\end{array}$ & $\begin{array}{c}0,157^{*} \\
(0,0821)\end{array}$ \\
\hline Autoeficacia ..................................... & $\begin{array}{l}1,208^{* * *} \\
(0,110)\end{array}$ & $\begin{array}{l}1,183^{* * *} \\
(0,111)\end{array}$ & $\begin{array}{l}1,180^{* * *} \\
(0,111)\end{array}$ & $\begin{array}{l}1,085^{\star * *} \\
(0,111)\end{array}$ & $\begin{array}{l}1,076^{* * *} \\
(0,112)\end{array}$ \\
\hline Temor al fracaso & $\begin{array}{l}-0,201^{* *} \\
(0,0944)\end{array}$ & $\begin{array}{l}-0,197^{* *} \\
(0,0946)\end{array}$ & $\begin{array}{l}-0,199^{* *} \\
(0,0948)\end{array}$ & $\begin{array}{l}-0,149 \\
(0,0973)\end{array}$ & $\begin{array}{l}-0,140 \\
(0,0976)\end{array}$ \\
\hline Exportador ......................................... & $\begin{array}{l}1,623^{* * *} \\
(0,145)\end{array}$ & $\begin{array}{l}1,582^{\star \star \star} \\
(0,147)\end{array}$ & $\begin{array}{l}1,571^{* * *} \\
(0,147)\end{array}$ & $\begin{array}{l}1,235^{\star * *} \\
(0,150)\end{array}$ & $\begin{array}{l}1,231^{\text {***}} \\
(0,150)\end{array}$ \\
\hline Inmigrante $\ldots \ldots \ldots \ldots \ldots \ldots \ldots \ldots \ldots \ldots \ldots \ldots \ldots \ldots \ldots \ldots \ldots \ldots \ldots \ldots \ldots \ldots \ldots \ldots$ & & & $\begin{array}{c}0,376^{*} \\
(0,210)\end{array}$ & $\begin{array}{c}0,419^{*} \\
(0,215)\end{array}$ & $\begin{array}{c}0,431^{* *} \\
(0,216)\end{array}$ \\
\hline Alta expec. crecimiento ......................... & & & & $\begin{array}{l}1,643^{* * *} \\
(0,116)\end{array}$ & $\begin{array}{l}1,631^{\text {** }} \\
(0,117)\end{array}$ \\
\hline \multicolumn{6}{|l|}{ Nivel Regional } \\
\hline \% población inmigrante ....................... & & $\begin{array}{l}0,0761^{* * *} \\
(0,0200)\end{array}$ & $\begin{array}{l}0,0716^{\star * *} \\
(0,0203)\end{array}$ & $\begin{array}{l}0,0668^{* * *} \\
(0,0201)\end{array}$ & $\begin{array}{l}0,0640^{\star *} \\
(0,0296)\end{array}$ \\
\hline \% participación en PIB & & $\begin{array}{l}-0,00956^{* * *} \\
(0,00325)\end{array}$ & $\begin{array}{l}-0,00959^{* * *} \\
(0,00325)\end{array}$ & $\begin{array}{l}-0,0114^{* * *} \\
(0,00335)\end{array}$ & $\begin{array}{l}-0,0116^{* *} \\
(0,00560)\end{array}$ \\
\hline 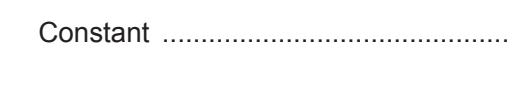 & $\begin{array}{l}-2,058^{* * *} \\
(0,152)\end{array}$ & $\begin{array}{l}-2,117^{\star * *} \\
(0,164)\end{array}$ & $\begin{array}{l}-2,123^{* * *} \\
(0,164)\end{array}$ & $\begin{array}{l}-2,270^{\star * *} \\
(0,174)\end{array}$ & $\begin{array}{l}-2,256^{\star * *} \\
(0,188)\end{array}$ \\
\hline 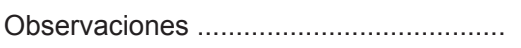 & 6,884 & 6,884 & 6,884 & 6,884 & 6,884 \\
\hline 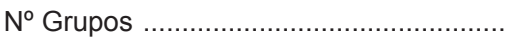 & & & & & 15 \\
\hline 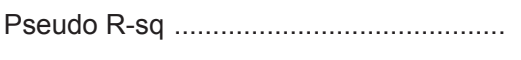 & 0,1096 & 0,1135 & 0,1141 & 0,1513 & \\
\hline \multicolumn{6}{|c|}{ NOTAS : Desviaciones estándar en paréntesis; $\left.{ }^{* * *} p<0,01,{ }^{* *} p<0,05,{ }^{*} p<0,1\right)$} \\
\hline
\end{tabular}




\section{GRÁFICO 1}

\section{PROBABILIDAD ESTIMADA DEL EMPRENDER INNOVADOR} (En \%)

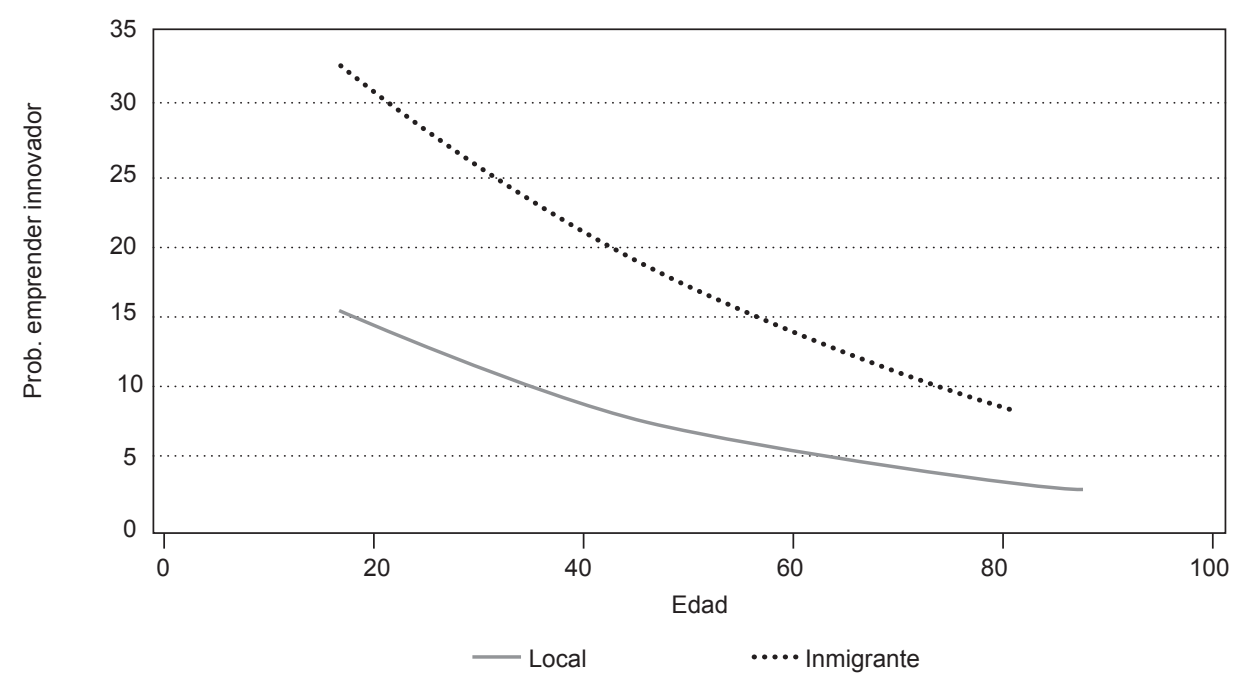

FUENTE: Elaboración propia en base a los efectos marginales de la regresión multinivel, columna 5 del Cuadro 3.

\section{Conclusiones}

Basándonos en la evidencia teórica y empírica previa, este estudio relaciona el vínculo entre innovación, inmigración y expectativas de crecimiento. Para eso, hemos analizado ciertos aspectos que suelen caracterizarse con el emprendedor que desarrolla un nuevo negocio que es innovador, diferenciándolo del emprendedor que crea uno que es en base a una estrategia de imitación. Los hallazgos apoyan y ratifican los beneficios potenciales de la inmigración y específicamente su relación con el desarrollo de emprendimientos innovadores y con altas expectativas de crecimiento. Los inmigrantes son frecuentemente descritos como una fuerza laboral que provee a la economía de asentamiento de una nueva configuración en el stock de capital humano (Ozgen et al., 2011).

Usando una muestra de 6.884 emprendedores, encontramos que la condición de inmigrante aumenta la probabilidad de un negocio con características innovadoras, ya sea en sus productos/servicios o en su proceso productivo. En este sentido, las mayores tasas de emprendimientos innovadores en términos relativos pueden atribuirse a inmigrantes emprendedores, lo que es relevante en el contexto de un país emergente como Chile, que ha atraído un creciente flujo de inmigrantes de países vecinos. Al respecto, es posible argumentar que, dado que la probabilidad de desarrollar emprendimientos innovadores está vinculada a la condición de inmigrante, un país que provea de una estructura institucional atractiva para inmigrantes podría indirectamente engrosar la actividad emprendedora local y la innovación.

Por otra parte, basándonos en argumentos a favor de los beneficios de la innovación, esperábamos que los emprendedores con altas expectativas de crecer tendrían mayores probabilidades de reportar un emprendimiento innovador. Los resultados confirman que aquellos que tienen altas expectativas de crecer son más propensos a presentar innovaciones en 
sus productos o procesos. Estos resultados son interesantes por diversas razones. Por un lado, nuestros resultados sugieren que los emprendedores tienden a ver en la innovación una orientación estratégica válida para poder cumplir con sus ambiciones de crecimiento (Aghion et al., 2001; Terjesen y Szerb, 2008; Stam y Wennberg, 2009). Si la innovación permite generar y preservar ventajas competitivas, la participación de mercado debiera aumentar sostenidamente, permitiendo el crecimiento del negocio. Sin embargo, es importante notar que las circunstancias en las que se siguen estrategias innovadoras parecen estar relacionadas con la forma en que el empresario interpreta sus aspiraciones en el negocio (Hessels et al., 2008). Esto puede revelar un sesgo en cómo los emprendedores dan sentido a sus percepciones del éxito a través de la innovación (Rosenbusch et al., 2011). Mientras que los emprendedores que tienen comparativamente menos expectativas de crecimiento reportan una menor probabilidad de innovar, el optimismo vinculado a la relación entre expectativas de crecimiento e innovación podría llevar a concluir que los emprendedores ven la innovación comparativamente mejor que imitar, en términos estratégicos, independientemente del contexto.

Nuestros resultados también proporcionan nuevas ideas sobre un debate emergente relacionado con el vínculo que hay con las características de quienes tienden a innovar. El análisis que se presenta en el Gráfico 1 sugiere que los emprendedores jóvenes, y que potencialmente tienen además menos experiencia, tendrían más probabilidades de reportar un emprendimiento innovador. Los emprendedores con más edad parecieran buscar minimizar su exposición al riesgo y reducir su incertidumbre con un negocio innovador, prefiriendo seleccionar una estrategia de imitación. Estas condiciones parecen minimizar también las expectativas que pueda haber del crecimiento del negocio. De esta forma, nuestros resultados revelan que el fomento de la inmigración, el incentivo para emprender joven y proporcionar un contexto adecuado que alimente las ambiciones de crecer son propulsores del emprendimiento innovador. Otro hallazgo relevante es que se observa que el nivel educacional no aumenta la probabilidad de desarrollar emprendimientos innovadores; la literatura muestra que mayores niveles de educación aumentan la probabilidad de emprender, pero el vínculo con los emprendimientos innovadores no es similar. Sí es relevante y positivo el capital social y la capacidad que los individuos se atribuyen para identificar oportunidades en el mercado.

Este estudio pretende establecer la relación entre la inmigración y las expectativas con el desarrollo de la innovación. La evidencia presentada en este estudio sugiere de manera concluyente que los inmigrantes y los emprendedores con grandes expectativas de crecer son más propensos a desarrollar emprendimientos innovadores en una economía emergente como la chilena, y por lo tanto un ecosistema integrado e inclusivo que permita igualdad de oportunidades para los inmigrantes puede ser un factor relevante para fomentar el emprendimiento y la innovación.

\section{Referencias bibliográficas}

[1] AGHION, P.; HARRIS, C.; HOWITT, P. y VICKERS, J. (2001). «Competition, Imitation and Growth with Step-by-step Innovation». The Review of Economic Studies, vol. 68, $n^{\circ} 3$, pp. 467-492.

[2] AGUINIS, H.; GOTTFREDSON, R. K. y CULPEPPER, S. A. (2013). «Best-practice Recommendations for Estimating Cross-level Interaction Effects Using Multilevel Modeling. Journal of Management, vol. 39, $n^{\circ} 6$, pp. 1.490-1.528.

[3] ALESINA, A. y LA FERRARA, E. (2005). «Preferences for Redistribution in the Land of Opportunities». Journal of Public Economics, vol. 89, n 5, pp. 897-931.

[4] AMASON, A. C.; SHRADER, R. C. y TOMPSON, G. H. (2006). «Newness and Novelty: Relating Top Management Team Composition to New Venture Performance». Journal of Business Venturing, vol. 21, $\mathrm{n}^{\circ}$ 1, pp. 125-148.

[5] AMORÓS, J. E.; VON BLOH, J.; LEVIE, J. y STERNBERG, R. (2016). "Transnational Diaspora Entrepreneurship (TDE) Meets Global Entrepreneurship Monitor (GEM): Preliminary Results of an Empirical Attempt to Measure TDE Between Countries». En 2nd International Conference on Migration and Diaspora Entrepreneurship, p. 43. 
[6] AUTIO, E.; PATHAK, S. y WENNBERG, K. (2013). «Consequences of Cultural Practices for Entrepreneurial Behaviors». Journal of International Business Studies, vol. 44, $\mathrm{n}^{\circ} 4$, pp. 334-362.

[7] BARON, R. A. y TANG, J. (2011). «The Role of Entrepreneurs in Firm-level Innovation: Joint Effects of Positive Affect, Creativity and Environmental Dynamism». Journal of Business Venturing, vol. 26, n 1, pp. 49-60.

[8] CHELLARAJ, G.; MASKUS, K. E. y MATTOO, A. (2005). «The Contribution of Skilled Immigration and International Graduate Students to US Innovation». World Bank Policy Research Working Paper, $\mathrm{n}^{\circ} 3.588$.

[9] DEWAR, R. D. y DUTTON, J. E. (1986). «The Adoption of Radical and Incremental Innovations: An Empirical Analysis». Management Science, vol. 32, no 11, pp. 1.422-1.433.

[10] DRUCKER, P. F. (1985). Innovation and Entrepreneurship Practices and Principles. AMACON.

[11] GORDON, I. R. y MCCANN, P. (2005). «Innovation, Agglomeration and Regional Development». Journal of ECOnomic Geography, vol. 5, n 5 , pp. 523-543.

[12] GREVE, H. R. y TAYLOR, A. (2000). «Innovations as Catalysts for Organizational Change: Shifts in Organizational Cognition and Search». Administrative Science Quarterly, vol. 45, n 1, pp. 54-80.

[13] GUNDAY, G.; ULUSOY, G.; KILIC, K. y ALPKAN, L. (2011). «Effects of Innovation Types on Firm Performance». International Journal of Production Economics, vol. 133, $\mathrm{n}^{\circ} 2$, pp. 662-676.

[14] GUNDRY, L. K. y WELSCH, H. P. (2001). «The Ambitious Entrepreneur: High Growth Strategies of Women-owned Enterprises». Journal of Business Venturing, vol. 16, $\mathrm{n}^{\circ} 5$, pp. 453-470.

[15] HART, D. M. y ACS, Z. J. (2011). «High-tech Immigrant Entrepreneurship in the United States». Economic Development Quarterly, vol. 25, n² 2, pp. 116-129.

[16] HESSELS, J.; VAN GELDEREN, M. y THURIK, R. (2008). «Entrepreneurial Aspirations, Motivations and their Drivers». Small Business Economics, vol. 31, n 3, pp. 323-339.

[17] HEUNKS, F. J. (1998). «Innovation, Creativity and Success». Small Business Economics, vol. 10, n 3, pp. 263-272.

[18] HUNT, J. y GAUTHIER-LOISELLE, M. (2010). «How Much does Immigration Boost Innovation?». American Economic Journal: Macroeconomics, vol. 2, n² 2, pp. 31-56.

[19] IRELAND, R. D. y WEBB, J. W. (2007). «A Multi-theoretic Perspective on Trust and Power in Strategic Supply Chains». Journal of Operations Management, vol. 25, n² 2, pp. 482-497.

[20] JACOBS, J. (1961). The Death and Life of the Great American Cities. Random House, New York. 1993. Modern Library Edition.

[21] JACOBS, J. (1969). The Economy of Cities. Random House, New York.
[22] KALLEBERG, A. L. y LEICHT, K. T. (1991). «Gender and Organizational Performance: Determinants of Small Business Survival and Success». Academy of Management Journal, vol. 1, pp. 136-161.

[23] KERR, W. R. (2010). «Breakthrough Inventions and Migrating Clusters of Innovation». Journal of Urban Economics, vol. 67, $\mathrm{n}^{\circ} 1$, pp. 46-60.

[24] KERR, W. R. (2013). US High-skilled Immigration, Innovation and Entrepreneurship: Empirical Approaches and Evidence, $\mathrm{n}^{\circ}$ 19.377. National Bureau of Economic Research.

[25] KIM, W. C. y MAUBORGNE, R. (1997). Value Innovation: The Strategic Logic of High Growth. Harvard Business School Pub.

[26] KLOMP, L. y VAN LEEUWEN, G. (2001). «Linking Innovation and Firm Performance: A New Approach». International Journal of the Economics of Business, vol. 8, n 3, pp. 343-364.

[27] KOCHHAR, R. (2015). A Global Middle Class is More Promise than Reality, $\mathrm{n}^{\circ} 641$. LIS Working Paper Series.

[28] KOELLINGER, P. (2008). «Why are Some Entrepreneurs More Innovative than Others?». Small Business Economics, vol. 31, $\mathrm{n}^{\circ} 1$, p. 21.

[29] KURATKO, D. F.; IRELAND, R. D.; COVIN, J. G. y HORNSBY, J. S. (2005). "A Model of Middle-Level Managers' Entrepreneurial Behavior». Entrepreneurship Theory and Practice, vol. 29, n 6 , pp. 699-716.

[30] MANDAKOVIC, V. y SEREY, T. (2017). Global Entrepreneurship Monitor: Reporte nacional de Chile 2016. Universidad del Desarrollo. Global Entrepreneurship Research Association.

[31] MARÉ, D. C.; FABLING, R. y STILLMAN, S. (2010). "Immigration and Innovation». Commissioned by the Economic Impacts of Immigration Research Programme of the Department of Labour. Wellington: Department of Labour.

[32] MONTI, D. J.; SMITH-DOERR, L. y MACQUAID, J. (2007). Immigrant Entrepreneurs in the Massachusetts Biotechnology Industry. Boston, MA: Immigrant Learning Center.

[33] NEVILLE, F.; ORSER, B.; RIDING, A. y JUNG, O. (2014). «Do Young Firms Owned by Recent Immigrants Outperform other Young Firms?». Journal of Business Venturing, vol. 29, no 1, pp. 55-71.

[34] OZGEN, C.; NIJKAMP, P. y POOT, J. (2011). «Immigration and Innovation in European Regions». Tinbergen Institute Discussion Paper, 2011-112/3.

[35] POOT, J. (2008). «Demographic Change and Regional Competitiveness: The Effects of Immigration and Ageing». International Journal of Foresight and Innovation Policy, vol. 4, $n^{\circ} 1-2$, pp. 129-145.

[36] RABE-HESKETH, S. y SKRONDAL, A. (2006). «Multilevel Modelling of Complex Survey Data». Journal of the Royal Statistical Society: Series A (Statistics in Society), vol. 169, $\mathrm{n}^{\circ} 4$, pp. 805-827. 
[37] RAUCH, A. y FRESE, M. (2007). «Let's put the Person Back into Entrepreneurship Research: A Meta-analysis on the Relationship Between Business Owners' Personality Traits, Business Creation and Success». European Journal of Work and Organizational Psychology, vol. 16, n 4, pp. 353-385.

[38] ROSENBUSCH, N.; BRINCKMANN, J. y BAUSCH, A. (2011). «Is Innovation Always Beneficial? A Meta-analysis of the Relationship Between Innovation and Performance in SMEs». Journal of Business Venturing, vol. $26, \mathrm{n}^{\circ} 4$, pp. 441-457.

[39] SAXENIAN, A. (1999). "Silicon Valley's new Immigrant Entrepreneurs». San Francisco: Public Policy Institute of California. vol. 32, pp. 1.991-2.005.

[40] STAM, E. y WENNBERG, K. (2009). "The Roles of R\&D in New Firm Growth». Small Business Economics, vol. 33, n 1, pp. 77-89.

[41] STEPHAN, P. E. y LEVIN, S. G. (2001). «Exceptional Contributions to US Science by the Foreign-born and Foreigneducated». Population Research and Policy Review, vol. 20, $\mathrm{n}^{\circ} 1$, pp. 59-79.
[42] STUART, T. E. (2000). «Interorganizational Alliances and the Performance of Firms: A Study of Growth and Innovation Rates in a High-technology Industry». Strategic Management Journal, pp. 791-811.

[43] STUEN, E. T.; MOBARAK, A. M. y MASKUS, K. E. (2012). "Skilled Immigration and Innovation: Evidence from Enrolment Fluctuations in US Doctoral Programmes». The Economic Journal, vol. 122, n 565, pp. 1.143-1.176.

[44] TERJESEN, S. y SZERB, L. (2008). «Dice Thrown from the Beginning? An Empirical Investigation of Determinants of Firm Level Growth Expectations». Estudios de Economía, vol. $35, \mathrm{n}^{\circ} 2$.

[45] WADHWA, V.; SAXENIAN, A.; RISSING, B. A. y GEREFFI, G. (2007). «America's New Immigrant Entrepreneurs: Part I. Duke Science, Technology and Innovation, Paper $n^{\circ}$ 23. Disponible en $h t t p: / / d x . d o i . o r g / 10.2139 / s s r n .990152$

[46] WADHWA, V.; SAXENIAN, A.; RISSING, B. A. y GEREFFI, G. (2008). «Skilled Immigration and Economic Growth». Applied Research in Economic Development, vol. 5, $n^{\circ} 4$. 